\title{
Support Vector Machine Based Bearing Fault Diagnosis for Induction Motors Using Vibration Signals
}

\author{
Don-Ha Hwang*, Young-Woo Youn*, Jong-Ho Sun*, Kyeong-Ho Choi**, Jong-Ho Lee*** \\ and Yong-Hwa Kim
}

\begin{abstract}
In this paper, we propose a new method for detecting bearing faults using vibration signals. The proposed method is based on support vector machines (SVMs), which treat the harmonics of fault-related frequencies from vibration signals as fault indices. Using SVMs, the cross-validations are used for a training process, and a two-stage classification process is used for detecting bearing faults and their status. The proposed approach is applied to outer-race bearing fault detection in threephase squirrel-cage induction motors. The experimental results show that the proposed method can effectively identify the bearing faults and their status, hence improving the accuracy of fault diagnosis.
\end{abstract}

Keywords: Bearing fault, Induction motor, Fault diagnosis, Vibration signal

\section{Introduction}

Induction motors are widely used as major electrical machines in a variety of industrial applications. Induction motor failures that are due to environmental stress and load conditions can result in severe damage to the motor itself as well as to motor-related industrial applications. It is well known that bearings are among the most common sources of motor faults in induction motors. Further, bearing faults represent $40-50 \%$ of the various types of induction motor faults $[1,2]$. The major source of bearing faults is damage on the inner or outer races of the bearing due to thermal or mechanical stresses $[2,3]$.

For bearing fault detection, various types of sensors and condition-monitoring systems have been employed [2-9]. Vibration measurements are commonly used to identify bearing faults [2, 5-9]. The amplitude at the bearing faultrelated frequency is used as the fault index in the vibration spectrum $[2,6,13]$. In addition, there are also harmonic frequencies in the vibration spectrum in motors with faulty bearings $[3,5,9]$. The spectral analysis of vibration signals is performed using the fast Fourier transform (FFT) method, which is the basic tool used together with some other approaches such as machine learning and statistical analysis.

To increase the accuracy of bearing fault detection, various machine learning and statistical analysis have been developed [10-13]. Neural-network-based fault diagnosis

$\dagger$ Corresponding Author: Dept. of Electronic Engineering, Yongin, Myongji University, Korea. (yongkim@mju.ac.kr)

* HVDC Research Division, Korea Electrotechnology Research Institute (KERI), Korea (\{dhhwang, ywyoun, jhsun\}@keri.re.kr)

** Dept. of Railroad Electricity, Kyungbuk College, Yeongju, Korea (ckh@kbc.ac.krr)

*** Dept. of Electronic Engineering, Gachon University, Seongnam, Korea. (jongho.lee@gachon.ac.kr)

Received: October 6, 2014; Accepted: February 4, 2015 has been proposed for rolling bearing faults using timefrequency domain vibration analysis [10]. The fuzzy classifier has been adopted to diagnose roller bearing faults using simple fuzzy rules and membership functions [11]. A support vector machine (SVM) was employed using time-domain and frequency-domain features for multiple faults diagnosis of induction motors [12]. Quadratic discriminant analysis and SVM have been used for multiple fault (air-gap eccentricity, bearing damages, and their combinations) detection using multiple sensors such as acoustic, vibration, and current sensors [13]. However, the effects of variations of the motor speed and fault severity have not been examined for SVM-based bearing fault detection $[12,13]$.

This paper considers bearing faults that are due to outerrace damages to the bearing in induction motors. In order to detect bearing faults, we implemented a two-stage diagnosis method for fault and fault-severity detections based on the FFT and SVMs. The first SVM classifier distinguishes faulty motors from healthy motors, while the second SVM classifier is used to discriminate between different bearing fault severities. The proposed fault detection method focuses on the spectra of vibration signals at fault-related frequency harmonics, and uses the values of the peak-to-mean ratio at harmonic frequencies as fault indices. Using the cross-validation for the SVM, the fault indices and SVM parameters are optimized from experiment results for different load conditions. The proposed diagnostic method can distinguish a faulty motor from a healthy motor with a probability of $100 \%$ of correct detection and a $0 \%$ likelihood of obtaining a false alarm under different load conditions. It can also discriminate between different severities with an average detection probability of $98.67 \%$ and a false alarm probability of $0 \%$ under different load conditions. 


\section{Outer-race Rolling Bearing Faults}

In this section, we present characteristic frequencies that are due to outer-race faults of rolling bearings and an experimental setup for rolling bearing fault detection.

\subsection{Characteristic frequencies}

The outer-race defect of rolling bearings induces a specific vibration frequency as shown below: $[2,9]$

$$
f_{O D}=\frac{N}{2} f_{r}\left(1-\frac{D_{B D}}{D_{P D}} \cos \varphi\right),
$$

where $N$ represents the number of rolling elements, $f_{r}$ is the mechanical rotational frequency, $D_{B D}$ is the rolling element diameter, $D_{P D}$ is the pitch diameter, and $\varphi$ is the contact angle. Moreover, when the defective area is large, the harmonics of $f_{O D}$ will also lead to the vibration spectrum as in $[3,5,10]$

$$
f_{O D, k}=k \times f_{O D}
$$

where $k=1,2,3, \cdots$ is the harmonic index.

In (1) and (2), the frequencies that are commonly used as a diagnostic measure for bearing fault detection in $[2,3,5$, $9,10]$, and vary depending on the load conditions. Table 1 lists the mechanical rotation frequency $f_{r}$ and the outerrace defect frequency $f_{O D}$ for test motors under different load conditions.

Table 1. Mechanical rotation frequency $f_{r}$ and outer-race defect frequency $f_{O D}$ for test motors

\begin{tabular}{c|c|c|c}
\hline Load condition $(\%)$ & No load & 50 & 100 \\
\hline$f_{r}(\mathrm{~Hz})$ & 29.99 & 29.8 & 29.66 \\
\hline$f_{O D}(\mathrm{~Hz})$ & 157.2924 & 156.2959 & 155.5616 \\
\hline
\end{tabular}

\subsection{Experimental setup}

Experimental tests were performed with $75-\mathrm{kW}$ squirrelcage induction motors, where the rated voltage is $3300 \mathrm{~V}$, the rated current is $16.3 \mathrm{~A}$, the supply frequency $f_{s}$ is 60 $\mathrm{Hz}$, the speed is $1780 \mathrm{rpm}$, and the number of poles is 4 . As shown in Fig. 1, the rolling bearing outer-race faults were simulated by making a hole in the outer race. This artificial fault cannot occur while a bearing is operating in a motor, but it is important to understand this fault in order to analyze the effects of bearing outer-race faults [3, 14]. For motor conditions, three types of test motors were used: a healthy motor, a motor with faulty bearing with an 8-mm hole, and a motor with faulty bearing with a 12-mm hole. In this paper, the two latter motors are labeled as "bearing 1 " and "bearing 2," respectively.

Fig. 2 shows the induction motor test system, which is composed of a test motor, a load motor, an inverter, and a

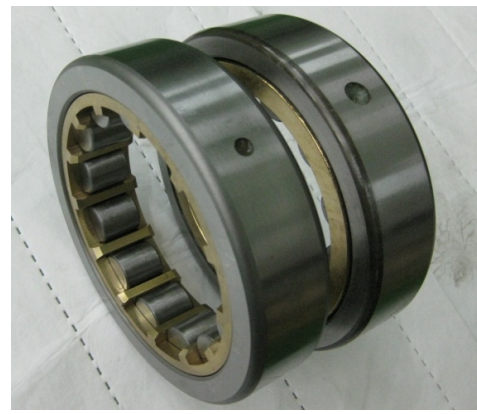

Fig. 1. Outer-race faults of roller bearings (bearing 1 and bearing 2).

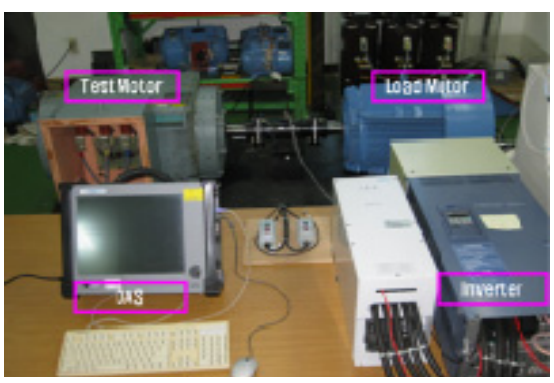

Fig. 2. Experimental setup.

data acquisition system (DAS) [15]. The test motor is equipped with a type NU318E roller bearing with $N=13$ rollers ( $D_{B D}$ and $D_{P D}$ are 28 and $145 \mathrm{~mm}$, respectively). An acceleration sensor (AS-022 from B\&K Vibro) is used, and is mounted on the test motor with $\varphi=0$. The inverter is connected to the load motor to control the load condition of the test motor. In the experimental tests, we considered for different load conditions of test motors, i.e., no load, $50 \%$, and $100 \%$ load. For the vibration measurement experiments, the discrete time signals $x_{S}[n]$ with $N_{S}=2^{22}$ were measured at a sampling frequency of $F_{S}=200 \mathrm{kHz}$ using the DAS, and the acquisition time was calculated as $T_{a c q}=20.972 \mathrm{~s}$.

\section{Proposed Diagnostic Method for Bearing Fault Detection}

To diagnose bearing faults using the spectra of the vibration signal, we propose a new detection method that is based on the SVM, which is a type of machine-learning technique based on the statistical learning theory. In the proposed approach, the objective of the SVM is to diagnose motor bearing faults using optimal fault indices from the fault-related harmonics of the most important components present in the spectrum of the vibration signals. In this section, we first introduce the SVM, emphasizing its use as a diagnostic tool for the vibration spectrum. Second, we propose an SVM-based two-stage diagnostic method that includes the feature calculation, feature selection, training, and classification, in order to detect the outer-race 
faults of rolling bearings.

\subsection{Background of SVM}

The basic idea that is introduced in this paper was thoroughly developed based on the statistical learning theory [16-19]. The basic SVM deals with two-class problems separating two classes by a hyperplane, which is defined by a number of support vectors.

In a linear separable case, there exists a separating hyperplane whose function is

$$
\boldsymbol{w} \cdot \boldsymbol{x}+b=0
$$

where the vector $\mathbf{w}$ defines the boundary, $\boldsymbol{x}$ is the input vector of dimension $d$, and $b$ is a scalar threshold. The optimal hyperplane can be obtained as follows [17]:

$$
\min J(\boldsymbol{w})=\frac{1}{2}\|\boldsymbol{w}\|^{2}, \text { subject to } y_{i}(\boldsymbol{w} \cdot \boldsymbol{x}+b) \geq 1,
$$

where $\|\boldsymbol{w}\|$ is the Euclidean norm of $\mathbf{w}, i=1, \cdots, l$ is the number of training sets, and labels $y_{i}=1$ and $y_{i}=-1$ are for positive and negative classes, respectively. The solution can be obtained by

$$
\boldsymbol{w}=\sum_{i=1}^{l} \alpha_{i} y_{i} \boldsymbol{x}_{i}
$$

where $\alpha_{i} \geq 0$ are Lagrange multipliers and $\boldsymbol{x}_{i}$ are support vectors obtained from training. After training, the decision function for the linear SVM is obtained as follows:

$$
f(\boldsymbol{x})=\operatorname{sign}\left(\sum_{i=1}^{l} \alpha_{i} y_{i}\left(\boldsymbol{x} \cdot \boldsymbol{x}_{i}\right)+b\right) .
$$

In a linear non-separable case, SVMs can create a hyperplane, which allows linear separation in the higher dimension, to perform a nonlinear mapping. The nonlinear mapping by the kernel function converts the input vector $\boldsymbol{x}$ from a $d$-dimensional space into a higher dimensional feature space. In nonlinear SVMs, kernel functions such as the polynomial, sigmoid, and radial basis functions (RBF) may be selected to obtain the optimal classification results [18]. In this study, the RBF kernel is used for nonlinear SVM and is defined by

$$
K(\boldsymbol{x}, \boldsymbol{y})=\exp \left(-\gamma\|\boldsymbol{x}-\boldsymbol{y}\|^{2}\right)
$$

where $\gamma>0$ is the RBF kernel parameter. For the nonlinear SVM, the decision function is obtained by

$$
f(\boldsymbol{x})=\operatorname{sign}\left(\sum_{i=1}^{l} \alpha_{i} y_{i} K\left(\boldsymbol{x}, \boldsymbol{x}_{i}\right)+b\right) .
$$

\subsection{Feature calculation}

The peak-to-average ratio (PR) has been proposed as an indicator to identify bearing faults in the spectrum [20]. The PR is defined as the sum of the peak values of the defect frequency and harmonics over the average value of the spectrum, and is defined as [20,21]

$$
P R=\frac{\sum_{k=1}^{K} P_{k}}{\frac{1}{J} \sum_{j=1}^{J} S_{j}},
$$

where $P_{k}$ is the amplitude of the maximum peak located at the frequency band that is centered at the $k$ th defect frequency harmonic, $f_{O D, k}$, with a bandwidth $B W, S_{j}$ is the amplitude at any frequency, $J$ is the number of points in the spectrum, and $K$ is the number of harmonics in the spectrum. In (9), the PR will contain the information at all harmonics contained in the vibration signal; however, only some of them will be significant depending on load rates. Therefore, the peak values of the defect frequency or harmonics are used as fault diagnostic indices, respectively, and are defined by

$$
P R_{k}=\frac{P_{k}}{\frac{1}{M} \sum_{j=1}^{M} W_{k, j}},
$$

where $k$ is the harmonic index, $W_{k, j}$ is the amplitude of frequency component at the frequency band centered at $f_{O D, k}$ with $B W$, and $M$ is the number of frequency-domain sample points in $B W$.

\subsection{Feature selection}

After feature calculation, a sequential forward search (SFS) creates candidate feature subsets using (2) for feature selection [13]. For each candidate feature subset, using $v$ fold cross-validation, the SFS examines the performance of a linear SVM when separating the data for healthy motors from those for faulty motors. In $v$-fold cross validation, the training set is divided into subsets of equal size and a subset is sequentially tested using the classifier trained on the remaining $v-1$ subsets. Based on experimental results, the motor speed can be calculated using the vibration signal [10], and therefore, feature selection gives the optimal feature subsets depending on variable load rates.

\subsection{Training and classification}

Based on the SVM algorithm, a two-stage classification is used to detect the motor fault and its severity. From the selected feature subset on the each load rate, the whole test data set is classified by the first SVM for the bearing fault 


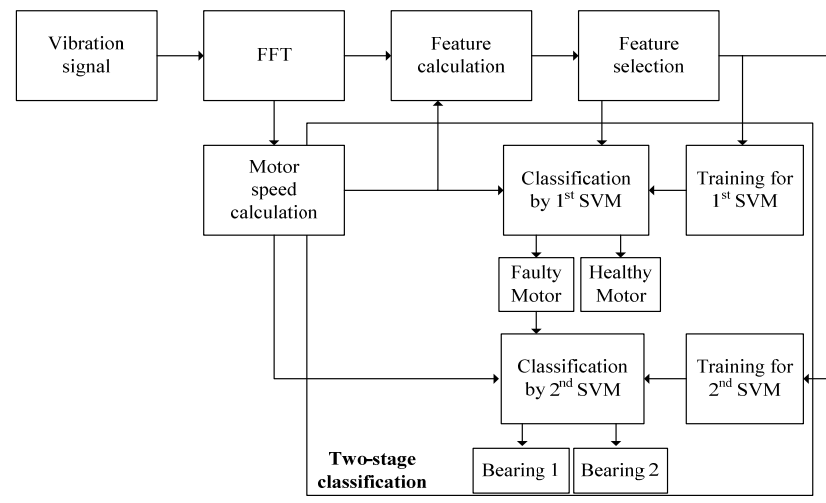

Fig. 3. Block diagram of the proposed fault diagnosis system.

detection. Then, some of the test data, which were classified as faulty motors by the first SVM, are classified by the second SVM to distinguish the severity of the bearing faults. The whole training set is used for the training of the first SVM, while the second SVM classifier exploits training data for faulty motors.

In the SVM training processes, to optimize the parameters $C$ for linear SVM or $\{C, \gamma\}$ for nonlinear SVM, we use the $v$-fold cross-validation to test all values of $C$ for the linear SVM or all of the pairs of $\{C, \gamma\}$ for the nonlinear SVM, where $C>0$ is the penalty parameter of the error term for SVMs, $v=5, C=\left\{2^{-15}, 2^{-14.9}, \cdots, 2^{15}\right\}$ for the linear SVM, and $C=\left\{2^{-10}, 2^{-9.5}, \cdots, 2^{10}\right\}$ and $\gamma=$ $\left\{2^{-10}, 2^{-9.5}, \cdots, 2^{10}\right\}$ for the nonlinear SVM [19]. Using the training set, $v$-fold cross-validation accuracies are obtained by the grid search, where the cross-validation accuracy is the percentage of data that are correctly classified. The values corresponding to the best crossvalidation accuracies are then selected. With the optimal parameters, the entire training dataset was trained again to define hyperplane for SVM classifiers. The process employed for the proposed fault diagnosis algorithm is illustrated in Fig. 3.

\section{Classification Results}

To validate the proposed method, we performed experimental tests using the rolling bearings of squirrelcage induction motors. The experiments were performed in the steady-state condition and the measured vibration signals were analyzed using the FFT. The Hanning window was used to minimize frequency leakage for the FFT [22] and the frequency resolution for the spectrum analysis was $\Delta f=0.0477 \mathrm{~Hz}$. For feature calculation in (9), we set the following values: $K=3$ and $J=N_{S} / 2$. The bandwidth $B W$ was $8 \mathrm{~Hz}$ for feature calculation in (10). Experiments were performed 50 times in each load condition.
For comparison purposes, the bearing fault detection with one feature was examined based on the signal detection theory [23]. Each fault index $A$ and the corresponding threshold parameter $A_{t h}$ were determined for each load condition using the combination criterion [15], [24], where $A \in\left\{P R, P R_{1}, P R_{2}, \cdots, P R_{5}\right\}$. The optimal fault index $A^{*}$ and optimal threshold $A_{t h}^{*}$ for the classifier with one feature is obtained by

$$
\left\{A^{*}, A_{t h}^{*}\right\}=\underset{\left\{A, A_{h l}\right\}}{\arg \max }\left\{P_{D, A}-P_{F A, A}\right\},
$$

where the detection probability $P_{D, A}$ and the false alarm probability $P_{F A, A}$ are defined as [23]

$$
\begin{gathered}
P_{D, A}=\operatorname{Pr}\left\{A>A_{t h} ; H_{1}\right\} \text { and } \\
P_{F A, A}=\operatorname{Pr}\left\{A>A_{t h} ; H_{0}\right\},
\end{gathered}
$$

respectively, $H_{1}$ is the bearing faulty motor hypothesis, and $H_{0}$ is the healthy motor hypothesis.

Fig. 4 shows the cumulative distributive functions (CDFs) of $P R$ and $P R_{1}$ under the no-load condition. For each fault index, the optimal threshold was determined based on the combination criterion. For the fault index $P R$,

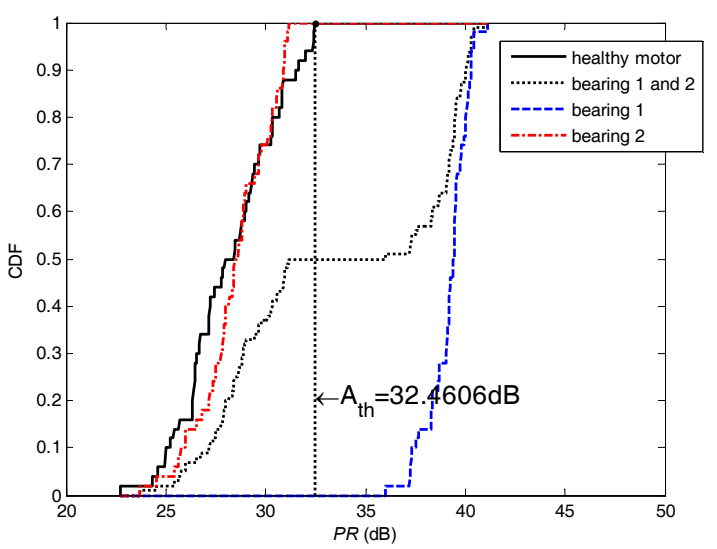

(a)

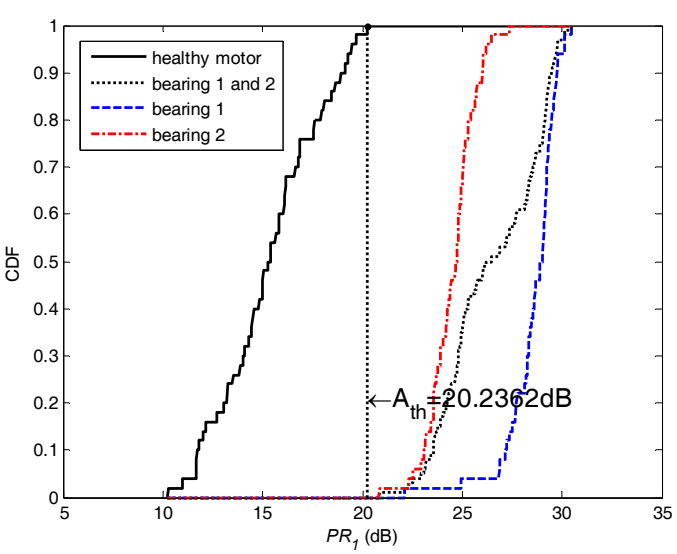

(b)

Fig. 4. CDFs for the fault detection with one feature under $0 \%$ load condition: (a) $P R$ and (b) $P R_{1}$. 
bearing 1 can be easily detected, but the difference between the healthy motor and bearing 2 is unclear. The fault index $P R_{1}$ is more evident than $P R$, and performs optimally when separating healthy motor from faulty motors. However, $P R_{1}$ does not perform optimally when discriminating between bearing 1 and bearing 2 under no-load conditions.

For the fault detection with one feature, $A^{*}$ and $A_{t h}^{*}$ are summarized in Table 2. The optimal fault index for noload and 50\% load conditions is $P R_{1}$. In particular, $P R_{3}$, which is obtained from the third harmonic of the vibration signal, is the optimal fault index under $100 \%$ load condition. For the bearing fault detection with one optimal feature, the detection probability decreases as the load rate increases. Figs. 5(a) and 5(b) show the CDFs of $P R_{1}$ and $P R_{3}$ under $50 \%$ and $100 \%$ load conditions, respectively.

Table 2. Fault detection performance for the classifier with one feature under different load condition

\begin{tabular}{c|c|c|c|c}
\hline $\begin{array}{c}\text { Load } \\
\text { condition (\%) }\end{array}$ & $A$ & $A_{t h}^{*}(\mathrm{~dB})$ & $P_{F A, A}$ & $\begin{array}{c}P_{D, A} \\
\text { (Bearings 1 and 2) }\end{array}$ \\
\hline 0 & $P R_{1}$ & 20.2362 & 0 & 1 \\
\hline 50 & $P R_{1}$ & 13.879 & 0.04 & 0.93 \\
\hline 100 & $P R_{3}$ & 10.471 & 0 & 0.92 \\
\hline
\end{tabular}

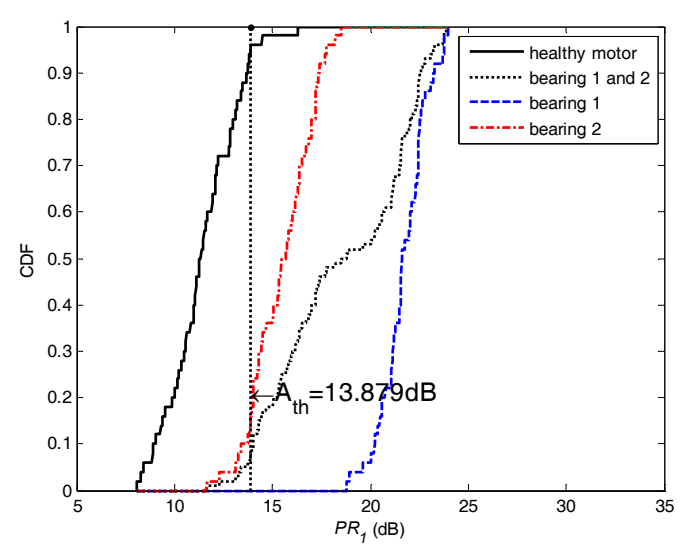

(a)

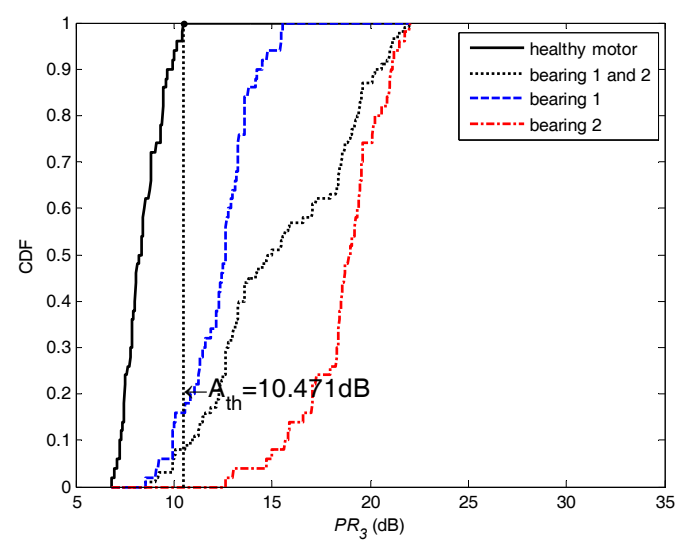

(b)

Fig. 5. CDFs for the fault detection with an optimal feature: (a) $P R_{1}$ under $50 \%$ load condition and (b) $P R_{3}$ under $100 \%$ load condition.
The amplitudes of $P R_{1}$ for bearing 2 are lower than those for bearing 1 under the $50 \%$ load condition, while the amplitudes of $P_{3}$ for bearing 2 are larger than those for bearing 1 under the $100 \%$ load condition. This is because the severity of the bearing faults induces the large harmonic of the fault frequency $[3,5,10]$; therefore, $P R_{3}$ is the dominant feature for bearing 2. As shown in this figure, fault detection with one optimal feature cannot guarantee an optimal performance for classifying a healthy motor, bearing 1 , or bearing 2 .

For the proposed scheme, using the SFS method, a subset of features $\left\{P R_{1}, P R_{2}, \cdots, P R_{5}\right\}$ is determined as the best feature set depending on the load rate. Fig. 6 illustrates the first classifier with the linear SVM using the best feature set $\left\{P R_{1}, P R_{3}\right\}$ on the $50 \%$ load rate. The parameter $C=2^{14.9}$ is determined from the fivefold crossvalidation analysis during the training process. Fig. 6 indicates that the healthy motors can be easily separated from the motors with faulty bearings (bearing 1 and bearing 2). For the $50 \%$ load condition, the proposed scheme is better than the previous fault detection method with one feature, as shown in Fig. 5(a).

For the $100 \%$ load rate, the best feature set can be obtained by $\left\{P R_{1}, P R_{3}\right\}$ using the SFS method for the first classifier with both the linear and nonlinear SVMs. As shown in Fig. 7(a), for the first classifier with the linear SVM, the detection probability is 0.98 and the false alarm probability is 0 , where $C=2^{2}$. Fig. 7(b) shows the performance of the first classifier with the nonlinear SVM, where the kernel parameters $C=2^{7}$ and $\gamma=2^{8}$ are used for the RBF kernel. The first classifier with the nonlinear SVM gives the optimal performance under a $100 \%$ load rate. Therefore, the proposed approach can correctly differentiate all motors with faulty bearings from healthy motors using the linear SVM under the $50 \%$ load condition, and nonlinear SVM under the $100 \%$ load condition. Table 3 summarizes the performance and parameters of the first classifier with linear and nonlinear SVMs. With the proper parameter $C$ and kernel parameter $\gamma$, the linear SVM obtains the

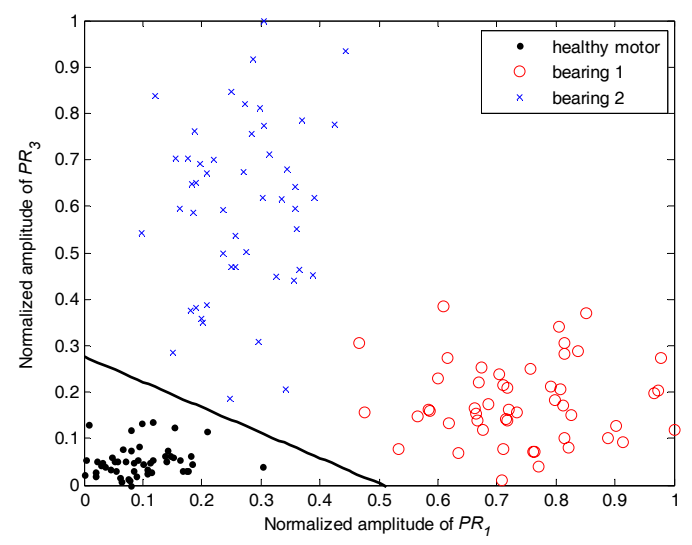

Fig. 6. The $1^{\text {st }}$ classifier with linear SVM for faulty motor detection under $50 \%$ load condition. 
Table 3. $1^{\text {st }}$ SVM classification results for fault detection

\begin{tabular}{c|c|c|c|c|c}
\hline SVM & $\begin{array}{c}\text { Load } \\
\text { condition(\%) }\end{array}$ & $\begin{array}{c}\text { Fault } \\
\text { indexes }\end{array}$ & $\begin{array}{c}\text { Kernel } \\
\text { parameters }\end{array}$ & $P_{F A}$ & $\begin{array}{c}P_{D} \text { (Bearings } \\
1 \text { and 2) }\end{array}$ \\
\hline Linear & 0 & $\left\{P R_{1}\right\}$ & $C=2^{1.1}$ & 0 & 1 \\
\hline Linear & 50 & $\left\{P R_{1}, P R_{3}\right\}$ & $C=2^{14.9}$ & 0 & 1 \\
\hline Linear & 100 & $\left\{P R_{1}, P R_{3}\right\}$ & $C=2^{2}$ & 0 & 0.98 \\
\hline $\begin{array}{c}\text { Non- } \\
\text { linear }\end{array}$ & 100 & $\left\{P R_{1}, P R_{3}\right\}$ & $\begin{array}{c}C=2^{7}, \\
\gamma=2^{8}\end{array}$ & 0 & 1 \\
\hline
\end{tabular}

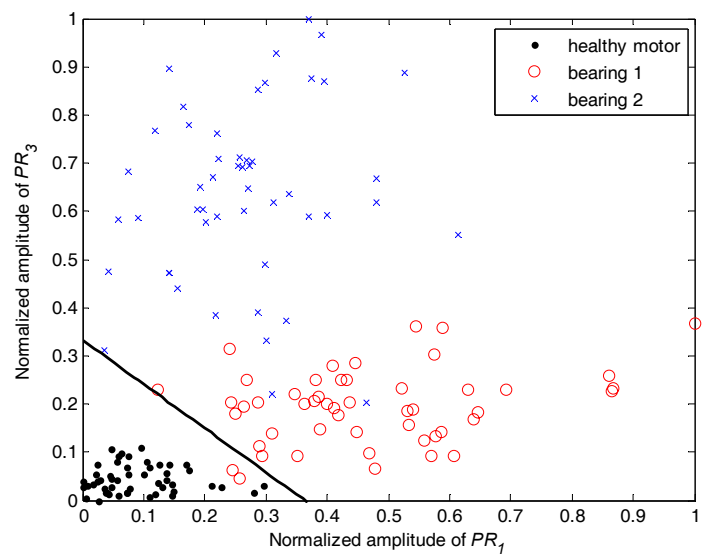

(a)

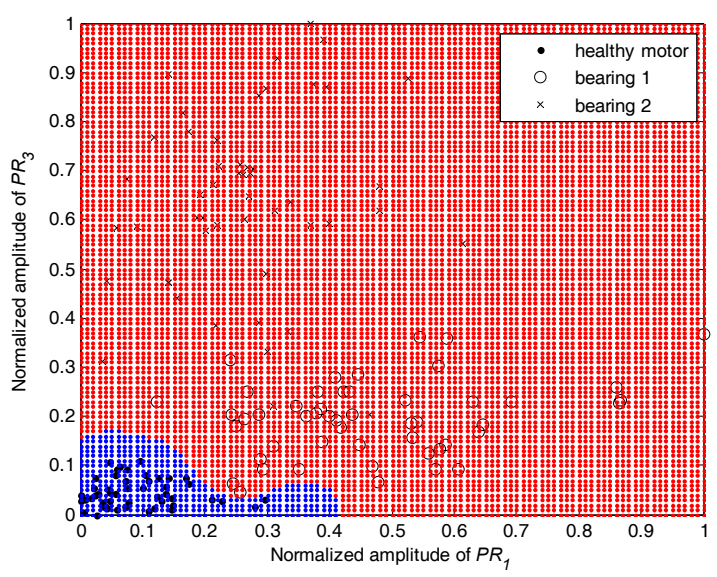

(b)

Fig. 7. The $1^{\text {st }}$ SVM classifier for faulty motor detection under 100\% load condition: (a) Linear SVM and (b) nonlinear SVM with RBF kernel.

optimal detection probabilities under $0 \%$ and $50 \%$ load conditions, and the nonlinear SVM gives the optimal detection probability under the $100 \%$ load condition. For linear and nonlinear SVMs, proper kernel parameter selection is important to obtain good classification results; therefore, a grid search of $C$ or $\{C, \gamma\}$ is needed to obtain the proper SVM parameters.

Finally, in Table 4, we present the fault classification using the $2^{\text {nd }}$ classifier for bearings 1 and 2 . It can be seen that almost all bearing fault types have been classified correctly under different load conditions. The feature set selected for the $2^{\text {nd }}$ classifier is based on the use of the
Table 4. $2^{\text {nd }}$ SVM classification results for fault type detection

\begin{tabular}{c|c|c|c|c|c}
\hline SVM & $\begin{array}{c}\text { Load } \\
\text { condition(\%) }\end{array}$ & $\begin{array}{c}\text { Fault } \\
\text { indexes }\end{array}$ & $\begin{array}{c}\text { Kernel } \\
\text { parameters }\end{array}$ & $P_{F A}$ & $P_{D}$ \\
\hline Linear & 0 & $\left\{P R_{3}\right\}$ & $C=2^{2.7}$ & 0 & 1 \\
\hline Linear & 50 & $\left\{P R_{1}, P R_{3}\right\}$ & $C=2^{-3.7}$ & 0 & 1 \\
\hline Linear & 100 & $\left\{P R_{1}, P R_{2}, P R_{3}, P R_{5}\right\}$ & $C=2^{4.6}$ & 0 & 0.96 \\
\hline $\begin{array}{c}\text { Non- } \\
\text { linear }\end{array}$ & 100 & $\left\{P R_{1}, P R_{2}, P R_{3}, P R_{5}\right\}$ & $\begin{array}{c}C=2^{-3}, \\
\gamma=2^{7.5}\end{array}$ & 0 & 0.96 \\
\hline
\end{tabular}

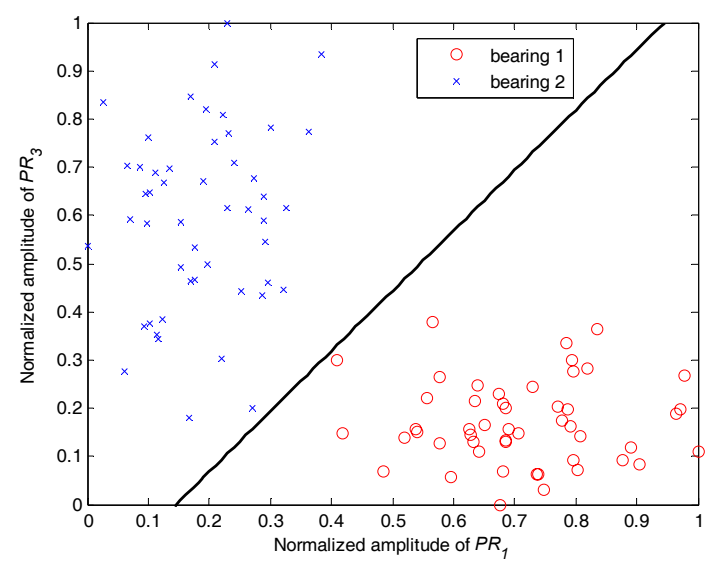

Fig. 8. The $2^{\text {nd }}$ SVM classifier used to separate bearings 1 and 2 under $50 \%$ load condition.

data obtained from faults involving bearings 1 and 2. Fig. 8 shows that both bearings 1 and 2 are in two separate clusters with the feature set of $\left\{P R_{1}, P R_{3}\right\}$ for the $50 \%$ load rate, where $C=2^{4.9}$. Compared to Fig. 6, the normalization set for the $2^{\text {nd }}$ classifier is different from that for the $1^{\text {st }}$ classifier, and therefore, the sample points in Fig. 8 are different from those in Fig. 6. Furthermore, the classification for bearing fault types on the $100 \%$ load rate has a high detection probability and low false alarm rate $\left(P_{D}=0.96\right.$ and $\left.P_{F A}=0\right)$.

\section{Conclusion}

In this paper, we proposed a new diagnosis method for rolling bearing faults in induction machines. The proposed method is based on the FFT and SVM methods, and consists of the two SVM classifiers to detect outer-race rolling bearing faults and their severity under different load conditions. The harmonics of fault-related frequency of vibration signal, which can be extracted from the vibration signal, serve as new bearing fault signatures. The optimization of the fault index subsets and hyperplanes was investigated using SVM cross-validation based on experimental data depending on the load conditions. An analysis of the experimental results shows that the proposed method has the higher detection probability and lower false alarm probability than the classifier with one 
feature under different load conditions. Experimental results show that the proposed two-stage classifier significantly improved the detection performance of bearing faults and their severity conditions. The proposed method will be useful in other bearing-related faults detections such as inner-race, cage, and ball faults modifying interested frequency bands.

\section{Acknowledgements}

This work was supported in part by the "Development of Motor Diagnosis Technology for the Electric Vehicle" project of Korea Electrotechnology Research Institute (KERI) and in part by the Human Resources Development program (No. 20134030200310) of the Korea Institute of Energy Technology Evaluation and Planning(KETEP) grant funded by the Korea government Ministry of Trade, Industry and Energy.

\section{References}

[1] W. T. Thomson and M. Fenger, "Current signature analysis to detect induction motor faults," IEEE Ind. Appl. Mag., vol. 7, no. 4, pp. 26-34, Jul./Aug. 2001.

[2] A. Bellini, F. Filippetti, C. Tassoni, and G.-A. Capolino, "Advances in diagnostic techniques for induction machines," IEEE Trans. Ind. Electron., vol. 55, no. 12, pp. 4109-4126, Dec. 2008.

[3] R. R. Schoen, T. G. Habetler, F. Kamran, and R. G. Bartheld, "Motor bearing damage detection using stator current monitoring," IEEE Trans. Ind. Appl., vol. 31, no. 6, pp. 1274-1279, Nov./Dec. 1995.

[4] W. Zhou, T. Habetler, and R. Harley, "Bearing fault detection via stator current noise cancellation and statistical control," IEEE Trans. Ind. Electron., vol. 55, no. 12, pp. 4260-4269, Dec. 2008.

[5] B. Li, G. Goddu, and M.-Y. Chow, "Detection of common motor bearing faults using frequency-domain vibration signals and a neural network based approach," Proc. of the 1998 American Control Conference, June 24-26, pp. 2032-2036, 1998.

[6] T. W. S. Chow and S. Hai, "Induction machine fault diagnostic analysis with wavelet technique," IEEE Trans. Ind. Electron., vol. 51, no. 3, pp. 558-565, Jun. 2004.

[7] J. R. Stack, R. G. Harley, and T. G. Habetler, "An amplitude modulation detector for fault diagnosis in rolling element bearings," IEEE Trans. Ind. Electron., vol. 51, no. 5, pp. 1097-1102, Oct. 2004.

[8] J. R. Stack, T. G. Habetler, and R. G. Harley, "Faultsignature modeling and detection of inner-race bearing faults," IEEE Trans. Ind. Appl., vol.42, no.1, pp. 61-68, Jan./Feb. 2006.

[9] C. Bianchini, F. Immovilli, M. Cocconcelli, R. Rubini, and A. Bellini, "Fault Detection of Linear Bearings in Brushless AC Linear Motors by Vibration Analysis," IEEE Trans. Ind. Electron., vol. 58, no. 5, pp. 16841694, May 2011.

[10] B. Li, C. Mo-Yuen, Y. Tipsuwan, and J. C. Hung, "Neural-network-based motor rolling bearing fault diagnosis," IEEE Trans. Ind. Electron., vol. 47, no. 5, pp. 1060-1069, Oct. 2000.

[11] V. Sugumaran, and K. Ramachandran, "Fault diagnosis of roller bearing using fuzzy classifier and histogram features with focus on automatic rule learning," Expert syst. Appl., vol. 38, no. 5, pp. 49014907, May 2011.

[12] A. Widodo, B. S. Yang, and T. Han, "Combination of independent component analysis and support vector machines for intelligent faults diagnosis of induction motors," Expert Syst. Appl., vol. 32, no. 2, pp. 299312, 2007.

[13] E. T. Esfahani, S. Wang, and V. Sundararajan, "Multisensor wireless system for eccentricity and bearing fault detection in induction motors," IEEE/ASME Trans. Mechatronics, vol. 19, no. 3, pp. 818-826, June 2014.

[14] L. Frosini, and E. Bassi, "Stator current and motor efficiency as indicators for different types of bearing faults in Induction motors," IEEE Trans. Ind. Electron., vol. 57, no. 1, pp. 244-251, Jan. 2010.

[15] Y.-H. Kim, Y.-W. Youn, D.-H. Hwang, J.-H. sun, and D.-S. Kang, "High-resolution parameter estimation method to identify broken rotor bar faults in induction motors," IEEE Trans. Ind. Electron., vol. 60, no. 9, pp. 4103-4117, Sept. 2013.

[16] V. N. Vapnik, The Nature of Statistical Learning Theory, New York:Springer, 1999.

[17] C. J. C. Burges, "A Tutorial on Support Vector Machines for Pattern Recognition," Data mining and Knowledge Discovery, vol. 12, pp.121-167, 1998.

[18] B. Scholkopf and A. J. Smola, Learning with Kernels: Support Vector Machines, Regularization, Optimization, and Beyond, The MIT Press, 2002.

[19] C. W. Hsu, C. C. Chang and C. J. Lin, "A Practical Guide to Support Vector Classification", 2007. [Online], Available: http://www.csie.ntu.edu.tw/ cjlin/libsvm.

[20] J. Shiroishi, Y. Li, S. Liang, T. Kurfess, and S. Danyluk, "Bearing condition diagnostics via vibration and acoustic emission measurements," Mechanical Systems and Signal Processing, vol. 11, no. 5, pp. 693-705, 1997.

[21] Y. H. Kim, A. C. C. Tan, J. Mathew, and B. S. Yang, "Condition monitoring of low speed bearings: A comparative study of the ultrasound technique versus vibration measurement," Proc. of WCEAM 2006, pp. 182-191, Jul. 2006.

[22] J.-H. Jung, J.-J. Lee, and B.-H. Kwon, "Online diagnosis of induction motors using MCSA," IEEE Trans. Ind. Electron., vol. 53, no. 6, pp. 1842-1852, 
Dec. 2006.

[23] S. M. Kay, Fundamentals of Statistical Signal Processing: Detection Theory. Englewood Cliffs, NJ: Prentice-Hall, 1993.

[24] W. Peterson, T. Birdsall, and W. Fox, "The theory of signal detectability," Proc. IRE Prof. Group Inf. Theory, vol. 4, no. 4, pp. 171-212, Sep. 1954.

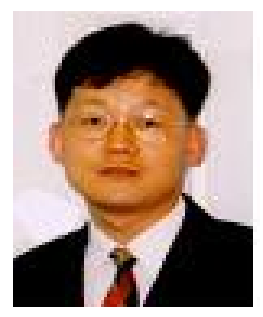

Don-Ha Hwang received B.S., M.S., and Ph.D. degrees in Electrical Engineering from Yeungnam University in 1991, 1993, and 2003, respectively. He is currently a principal researcher at Korea Electrotechnology Research Institute (KERI), Changwon, Korea. His main research interest are design, analysis, monitoring, and diagnosis of electric machines.

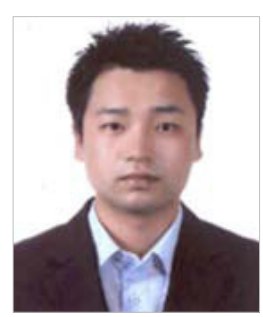

Young-Woo Youn received B.S. and M.S. degrees in Communication Engineering from Information and Communication University, Daejeon, Korea, in 2005 and 2007, respectively. He is currently a researcher at power apparatus research center at Korea Electrotechnology Research Institute (KERI), Changwon, Korea. His research interests are in condition monitoring and signal processing.

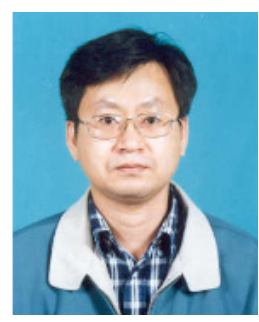

Jong-Ho Sun received B.S., M.S., and Ph.D. degrees in Electrical Engineering from Pusan National University in 1986, 1988, and 2001, respectively. Currently, he is a principal researcher at Korea Electrotechnology Research Institute (KERI), Changwon, Korea. His interests are diagnosis techniques for electric power equipments.

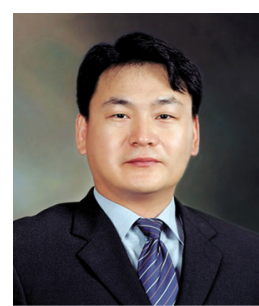

Kyeong-Ho Choi received B.S., M.S., and Ph.D. degrees in Electrical Engineering from Yeungnam University in 1991, 1995, and 2002, respectively. Currently, he is a professor in the departement of railroad electricity at Kyungbuk College, Korea.

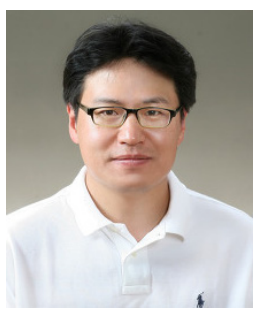

Jong-Ho Lee received the B.S. degree in electrical engineering and the M.S. and Ph.D. degrees in electrical engineering and computer science from Seoul National University, Seoul, Korea, in 1999, 2001, and 2006, respectively. From 2006 to 2008, he was a Senior Engineer with Samsung

Electronics, Suwon, Korea. From 2008 to 2009, he was a Postdoctoral Researcher with the Georgia Institute of Technology, Atlanta, GA, USA. From 2009 to 2012, he was an Assistant Professor with the Division of Electrical Electronic and Control Engineering, Kongju National University, Cheonan, Korea. Since 2012, he has been with the faculty of the Department of Electronic Engineering, Gachon University, Seongnam, Korea. His research interests are in the area of wireless communication systems and signal processing for communication with current emphasis on multiple antenna techniques, multi-hop relay techniques, physical layer security, and full-duplex wireless.

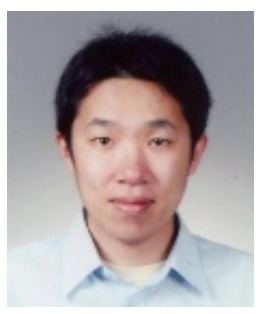

Yong-Hwa Kim received a B.S. in electrical engineering in 2001 from Seoul National University, Seoul, Korea, and a Ph.D. in electrical and computer engineering from Seoul National University, Seoul, Korea, 2007. From 2007 to 2011, he was a senior researcher with the Korea Electrotechnology Research Institute (KERI), Geonggi-do, Korea. From 2011 to 2013, he was an assistant professor at the Division of Maritime Electronic and Communication Engineering, Mokpo National Maritime University, Korea. Since March 2013, he has joined the faculty with the Department of Electronic Engineering at Myongji University, Korea. His general research interests include communication systems, motor drives and diagnosis, and digital signal processing. Currently, he is particularly interested in communications and digital signal processing for Smart Grid. 